\title{
System design and analysis of UAV-assisted BLE wireless sensor systems
}

\author{
Mikhail Komarov $^{1,2}$ and Dmitri Moltchanov ${ }^{2}$ \\ 1 School of Business Informatics, Faculty of Business and Management \\ National Research University Higher School of Economics, Moscow, Russia \\ mkomarov@hse.ru \\ 2 Department of Communications Engineering, \\ Tampere University of Technology, Tampere, Finland, \\ \{mikhail.komarov,dmitri.moltchanov\}@tut.fi
}

\begin{abstract}
Inefficiency of wireless sensor networks (WSN) in terms of the network lifetime is one of the major reasons preventing their widespread use. To alleviate this problem different data collection approaches have been proposed. One of the promising techniques is to use unmanned aerial vehicle (UAV). In spite of several papers advocating this approach, there have been no system designs and associated performance evaluation proposed to date. In this paper, we address this issue by proposing a new WSN design, where UAV serves as a sink while Bluetooth low energy (BLE) is used as a communication technology. We analyze the proposed design in terms of the network lifetime and area coverage comparing it with routed WSNs. Our results reveal that the lifetime of the proposed design is approximately two orders of magnitude longer than that of the routed WSNs. Using the tools of integral geometry we show that the density of nodes to cover a certain area is approximately two times more for routed WSNs compared to our design.
\end{abstract}

Keywords: sensor networks, BLE, UAV, system design, performance

\section{Introduction}

A wireless sensor network is defined as a collection of devices capable of sensing environmental parameters, connected in a network and collectively delivering the collected data to a certain location. As a result of more than a decade of investigations, the challenges and requirements of modern WSNs are well understood $[1,13]$. There are already a number of complete solutions available on the market. At the same time, in spite of significant market push from the vendors WSNs are still not widely deployed. One of the major reasons is inefficiency of such systems in terms of the network lifetime.

Uneven energy consumption is one of the reasons for short WSN lifetimes. The root cause of this phenomenon is networking of nodes. In practical deployments there are only few locations, where sinks can be positioned. In this case there is always a set of nodes that are more involved in packets routing and 
forwarding. Since the lifetime of a network is defined as the time till there is no path to the sink we see that uneven energy consumption places severe constraints on lifetime. Over the last decade researchers addressed this problem identifying a number of feasible solutions such as multi-path routing, clusterization, data aggregation, in-network data processing, etc. However, none of those are general enough to be applicable to any arbitrary deployment [3]. On top of this, the routed principle of WSNs adds to this problem. Indeed, networking mechanisms such as neighbor discovery, connectivity and topology maintenance, routing and packet forwarding require substantial amount of energy $[4,2]$.

One way to avoid unequal energy consumption is to get rid of networking. Mobile sinks may allow to achieve this goal. To avoid human involvement the collection of data must be completely automatic. The obvious choice would be to use unmanned aerial vehicles (UAV), particularly, quadrocopters, also known as drones. UAVs are a new technology at the "rapid improvement" phase of the $S$-curve of technology development cycle. As improvements are made, drones are becoming more agile, autonomous, power efficient and safe. The idea of using UAVs for data collection in WSNs is not new. Particularly, the authors in [12] advocated the use of UAV to interconnect sparse WSN clusters. The use of UAV as a mobile WSN node for emergency applications has been suggested in [8]. The authors in [11] proposed to use UAV for charging and deploying WSN nodes. Nevertheless, to date, no detailed investigations of such solutions and/or their comparison with conventional routed WSN designs have been performed.

We propose a new UAV-assisted solution for data collection in WSNs. We optimize performance of single hop communications between a sensor node and UAV in terms of optimal altitude and and flying speed. We also compare lifetimes, coverage and required density of nodes of our solution with those of routed WSN designs. Using the tools of integral geometry and random graphs theory we show that (i) the lifetime of our design is 10-18 times longer compared to routed WSNs operating using the same BLE technology, (ii) the required density of nodes for environment monitoring is approximately two times smaller even when sensing range coincides with the communications range. These two properties make UAV-assisted WSN design an attractive option for many applications including smart agriculture, forest fire monitoring, etc.

The rest of the article is organized as follows. Section 2 provides state-ofthe-art in UAV and short-range wireless communications backing up the choice of drones and BLE for our design. System parameters are optimized in Section 3 . We analytically compare performance of routed WSN solution and the proposed one in terms of network lifetime, coverage and connectivity in Section 4. Conclusions are drawn in the last section.

\section{Technological choices}

\subsection{Communication technology}

There are several options to consider as a candidate wireless technology for single hop wireless sensor systems. The comparison of these technologies is shown in 
Table 1. Comparison of low power communications technologies.

\begin{tabular}{|l|l|l|l|l|l|}
\hline Metric & $\mathrm{PPB} \mu \mathrm{W} /$ bit & Range, $\mathrm{m}$ & Rate, Mbps & Delay, ms & $\mathrm{PPC}, \mathrm{mA}$ \\
\hline \hline Bluetooth & 0.05 & 30 & 2.1 & 20 & 100 \\
\hline BLE & 0.153 & 50 & 0.3 & $3-6$ & 12.5 \\
\hline ANT+ & 0.71 & 30 & 0.002 & $<1$ & 17 \\
\hline ZigBee & 185 & 100 & 0.1 & 20 & 40 \\
\hline Wi-Fi & 0.005 & 150 & 6 & 1.5 & 50 \\
\hline Nike+ & 2.48 & 10 & 0.0003 & 1000 & 12.3 \\
\hline
\end{tabular}

Table 1, where PPC stands for peak power consumption, PPB refers to powerper-bit. In this paper we advocate the use of recently standardized BLE. BLE is an evolution of Bluetooth for state transmission systems, i.e. sensor data. Table 2 summarizes the most important improvements introduced in BLE compared to Bluetooth. Modifications affected the critical features including physical layer modulation, communications protocols, application interface and security. One important improvement is the use of Gaussian Frequency Shift Keying with larger modulation index, $0.45-0.55$ instead of $0.25-0.35$ in Bluetooth. Recall, that the modulation index in GFSK affects the wideness of the signal spectrum. This allowed to increase the communications range of the BLE technology while using the same amount of emitted power. Further, significant changes has been introduced to the communication protocol limiting almost all basic parameters. Targeting state applications the maximum packet size was reduced to 27 bytes while the acknowledgement packet size was reduced from 100 bytes to 12 bytes. The authentication in BLE is performed for each packet favoring single packet transactions. Encryption is performed using AES-128. Of special importance is the reduction in the connection establishment time allowing to perform it in just $3 \mathrm{~ms}$ and completing the transfer of one packet in less than a second. Recalling compatibility with Bluetooth, these features make BLE an excellent choice for power-constrained single-hop wireless sensor systems.

\subsection{UAV technology}

Over the last decade the UAV technology has advanced beyond the domain of military sponsored projects. Civilian applications are becoming more and more common. A whole new class of drones has evolved over the last decade - the Micro Air Vehicles (MAV). It encompasses a whole range of miniature, flying vehicles that have been around for a while now. Recently, multi-rotor UAVs, also referred to as drones, have attracted significant attention. These devices use three or more vertically aligned engines and movement is achieved by creating a difference in thrust on motors on the opposite sides of the frame resulting the UAV tilting and creating sideways acceleration. The thrust regulation needs to be very precise and cannot be done without a microcontroller. To perform basic operations, the microcontroller needs sensors including gyroscope and accelerometer.

The first drones were built around Arduino platform, so they already had a surplus of processing power and are compatible with many more sensors than 
Table 2. Comparison of Bluetooth and BLE.

\begin{tabular}{|l|l|l|}
\hline Parameter & Bluetooth & BLE \\
\hline \hline Channels & 79 & 40 \\
\hline Modulation/index & GFKP/0.35 & GFSK/0.55 \\
\hline Tolerable pathloss & $90 \mathrm{~dB}$ & $95 \mathrm{~dB}$ \\
\hline Range & $30 \mathrm{~m}$ & $50 \mathrm{~m}$ \\
\hline ACK length & $100 \mathrm{~B}$ & $12 \mathrm{~B}$ \\
\hline Max packet size & $1021 \mathrm{~B}$ & $27 \mathrm{~B}$ \\
\hline CRC length & 2B & $3 \mathrm{~B}$ \\
\hline Encryption & Safer+ & AES-128 \\
\hline Authentication & Once & Every packet \\
\hline Connection states & Inquiry/Page/Connected & Advertizing \\
\hline Connection time & $20 \mathrm{~ms}$ & 3ms \\
\hline
\end{tabular}

just the essentials. Achieving autonomous flight was just a matter of adding additional sensors and programming the software to use them. This trend still continues today and, as the sensors grow in numbers, so does the processing power needed to make effective use of them. As of today, widely available drones use dedicated Flight Management Units (FMUs) that come with all the basic sensors integrated on one board and a further support for the most common sensor buses like CAN or I2C.

A commercial UAV include systems for precise navigation, collision protection, home landing and programmable flying route. With maximum flying speed of $15 \mathrm{~m} / \mathrm{s}(54 \mathrm{~km} / \mathrm{h})$ and time of $30-90 \mathrm{~m}$ an UAV can fly up to $79.2 \mathrm{~km}$ in a single run. Assuming that an UAV carries a BLE device with communication range of $50-100 \mathrm{~m}$, flies in zig-zag way to scan sensors on monitored area, the coverage area of a single flight is $5.5-16.0 \mathrm{~km}^{2}$.

\subsection{System design overview}

The system design is illustrated in Fig. 1. Instead of keeping sink node stationary and gathering data by multi-hop routing, we make it mobile and apply BLE technology to further reduce power consumption of nodes. There are multiple advantageous features of using UAVs for data collection. First, flexibility of movement allows to freely optimize trajectories collecting data from the whole network or only from a part of it. Secondly, the movement speed is high allowing to cover the large areas of interest. Taking into account the recent progress in UAVs, the flight navigation could be made completely automatic using GPS. In this mode, an UAV can be configured to follow a predetermined route to collect sensors' data then come back to a ground station to transfer data and recharge battery. A group of UAVs can be used in combination with a ground station or adjacent stations to collect sensors' data in one sensing area or in different ones.

The proposed WSN system is based on single-hop data transmission approach where a UAV carries a sink node, BLE master, and fly over the monitored area to collect data from deployed sensor nodes, BLE slaves. Sensor and sink 


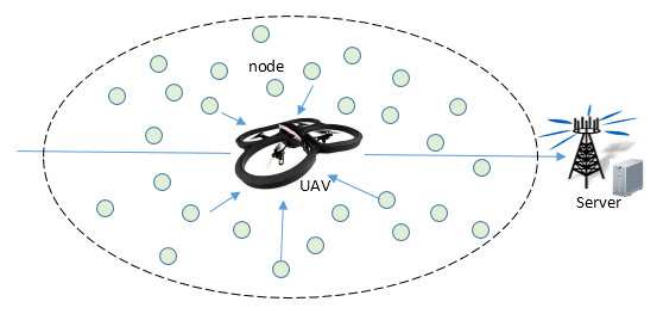

Fig. 1. Proposed WSN data collection mechanism.

nodes communicate with each other using BLE. Sink node continuously scan for BLE slave nodes to establish connections and exchange data. After data collection, UAV comes back to ground station. The system does not require time synchronization between UAV flying time and BLE slave wake up pattern.

\section{System design}

\subsection{Device discovery time}

To exchange data BLE device have to discover its neighbors. It starts with entering the advertising state and then proceeding with connection state. In advertising state the sensor node sends advertising packets over three designated channels $(37,38$, and 39). The scanner scans these channels continuously. The role of the scanner is performed by the UAV. When scanner discovers an advertiser, it sends a connection request packet to establish connection. Once replied, both devices enter the connection mode. While connected, advertiser becomes slave and scanner is designated as master.

The advertising interval, $T_{A}$, consists of a static interval $T_{A, 0}$ and a random part $\rho$, where $\rho$ is uniformly distributed between 0 and $10 \mathrm{~ms}, T_{A, 0}$ is chosen in between $20 \mathrm{~ms}$ and $10 \mathrm{~s}$. There are two time intervals defined for the scanner, scanning interval $T_{S}$, chosen in between $20 \mathrm{~ms}$ and $10 \mathrm{~s}$, and the scanning event time $d_{s}$ chosen in between 0 and $T_{S}$. The channel interval corresponds to the time to send 38 bytes of advertizing packet and equals to $446 \mu \mathrm{s}$. The interval between sending advertizing packets within a single adverstizing event is $d_{c h}=150 \mu \mathrm{s}$.

Let $T_{d i s}$ be the discovery time. Analyzing Fig. 2 we see that the discovery is successfull if $d_{A}<T_{d i s}<T_{A}+d_{A}$ implying that the upper bound on the discovery time is $10.24+0.01+0.000446 \approx 10.25 \mathrm{~s}$ while the minimum is exactly the time to transmit 38 bytes of data, $446 \mu$ s.

\subsection{Connection time}

Having received an advertising packet, the scanner sends a connection request packet after waiting for distributed interframe spacing time (DIFS, $d_{I F S}$ ). This 


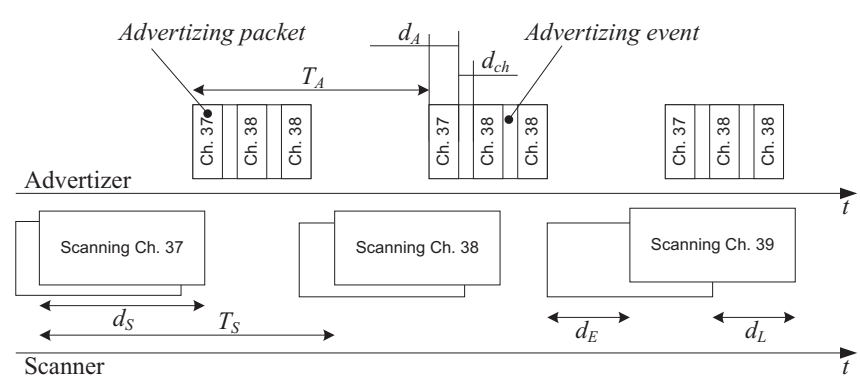

Fig. 2. Time diagram of advertising/scanning phase in BLE.

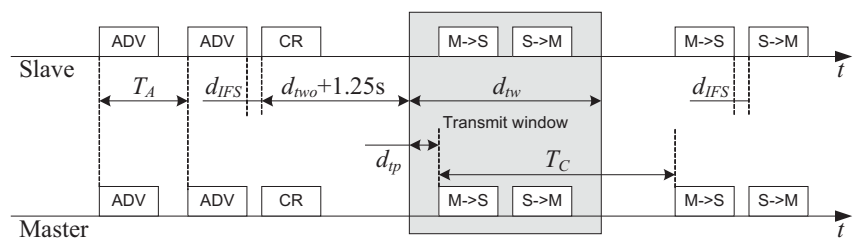

Fig. 3. Time diagram of connection phase in BLE.

packet contains two parameters, transmitWindowOffset, $d_{t w o}$, and transmitWin$d o w, d_{t w}$. According to our assumptions all the data can be transmitted in just one packet. Fig. 3 illustrates the connection phase between master and slave.

Denoting the connection time by $T_{c o n}$ we see that

$$
T_{\text {con }}=d_{I F S}+1.25 m s+d_{t w o}+d_{t w}
$$

where $d_{I F S}=150 \mu \mathrm{s}, 0<d_{t w o}<T_{C}, 1.25 \mathrm{~ms}<d_{t w}<10 \mathrm{~ms}, T_{C}$ is the connection interval which could be tuned between $7.5 \mathrm{~ms}$ and $4 \mathrm{~s}$. Thus, we see that connection time is bounded by

$$
d_{I F S}+1.25 m s \approx 1.4 m s<T_{\text {con }}<4.0114 s . \approx d_{t w o}+1.25 s+d_{t w} .
$$

In the proposed system, an UAV scans for advertising packets continuously while the BLE module of a sensor node enter advertizing state after each wake up. The bounds on the time to transfer data, $T=T_{d i s}+T_{c o n}$, is

$$
446 \mu s+1.4 m s \approx 2 m s<T<14.262 s=10.250446 s+4.0114 s .
$$

implying that remaining in the coverage of a sensor node for at least $14 \mathrm{~s}$ will guarantee exchange of data.

\subsection{Optimal UAV altitude and speed}

Consider a sensor node positioned on the ground and UAV flying over. Assume that the radio of a sensor node is omnidirectional forming a half-sphere around 


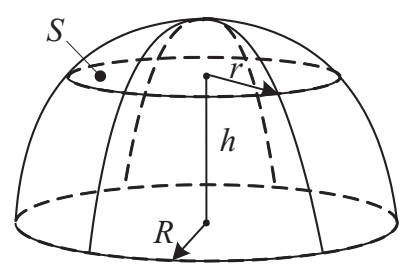

Fig. 4. An illustration of the UAV flying over a BLE sensor node.

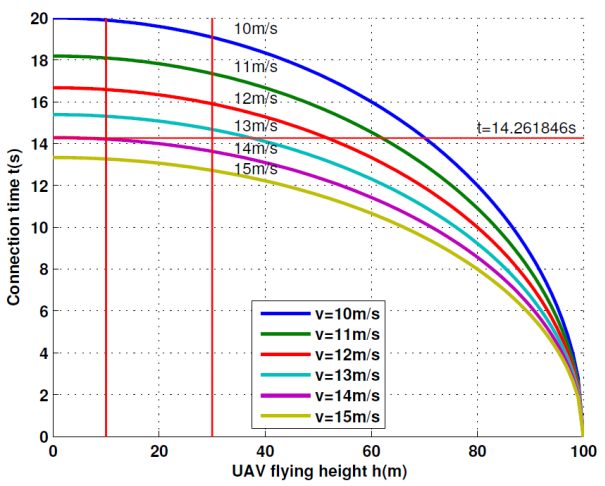

Fig. 5. UAV altitude and transaction time for different UAV speeds.

the node's position as shown in Fig. 4. The typical speed of UAV varies in the range of $30-45 \mathrm{~km} / \mathrm{h}, 10-15 \mathrm{~m} / \mathrm{s}$. BLE range, $R$, is assumed to be upper bounded by $100 \mathrm{~m}$. According to the previous section the connection time is upper bounded by approximately $14 \mathrm{~s}$. We assume that when UAV crosses the half-sphere shown in Fig. 4 it goes through the center of the cutting plane to increase its chances to establish connection and collect the data from the sensor.

Analyzing Fig. 4, we see that the maximum altitude of UAV is given by

$$
h=\sqrt{R^{2}-\left(v T_{T} / 2\right)^{2}},
$$

where $T_{T}=T_{d i s}+T_{c o n}$ is the transaction time, $v$ is the speed of UAV.

Keeping UAV flying speed low, UAV may have enough time to exchange data with sensor node but the coverage range of UAV decreases. To increase UAV velocity, we need to decrease the altitude. The dependencies between UAV altitude, speed and connection time is shown in Fig. 5.

\subsection{Transmission power requirements}

The commercial BLE chipsets (CC2541, CC2540, [5]) are produced with programmable output power that could be adjusted over a wide range. Relying on the estimated UAV flying parameters the task is to determine the minimum transmission power needed to establish connection and exchange the data [9]. 
Since the proposed system is applied for monitoring flat area with line-ofsight between UAV and sensor node, the free space path loss propagation model can be applied. Using Friis equation in the form

$$
P_{R x}=P_{T x}+G_{T}+G_{R}-20 \log _{10}(d)-20 \log _{10}(f)+147.55,
$$

we derive $P_{T x}$, where $P_{R x}$ is the minimum power at the receiver set to $-90 \mathrm{dBm}$, $P_{T x}$ is the transmission power we are looking for, $G_{T}=G_{R}=6 \mathrm{dBi}$ are antenna gains at the transmitter and receiver, respectively, $d$ is transmission range in kilometers and $f=2.4 \mathrm{GHz}$ is the operating frequency.

Fig. 6 shows the relation between the required transmission power and transaction time when UAV altitude varies between $10 \mathrm{~m}$ and $20 \mathrm{~m}$. The transmission power in the proposed WSN design is extremely low ranging from $-224.946 \mathrm{dBm}$ to $-207.702 \mathrm{dBm}$.

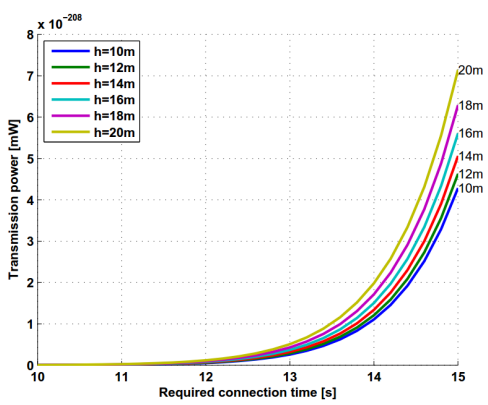

(a) Power in $\mathrm{mW}$

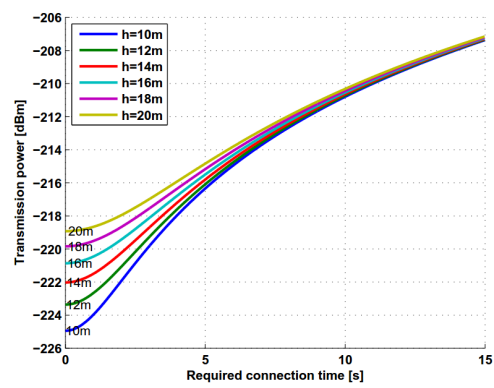

(b) Power in $\mathrm{dB}$

Fig. 6. The required transmission power for different transaction times.

\section{Performance analysis and comparison}

\subsection{Network lifetime}

In the proposed design all the nodes spend equal amount of power. Consider the power required to perform a single transaction. The power consumption of the radio states of advertising and connection events is provided in [5]. Based on these data, the power consumption for advertising and connection events are

$$
P_{A}=\frac{U \sum_{i} I_{i} t_{i}}{\sum_{i} t_{i}}=32.759, \quad P_{C}=\frac{U \sum_{i} I_{i} t_{i}}{\sum_{i} t_{i}}=24.762,
$$

leading to the overall consumption per transaction $P=57.521 \mathrm{~mW}$, that is, $19.173 \mathrm{~mA}$ in $6.92 \mathrm{~ms}$. Assuming that sensor nodes typically use a coin-cell CR2032 battery with capacity $225 \mathrm{mAh}$, a single node can work continuously during 20.605 hours. If we set the device to wake up periodically in each $10 \mathrm{~s}$, it can last for approximately 2020 days or around 5.53 years. 


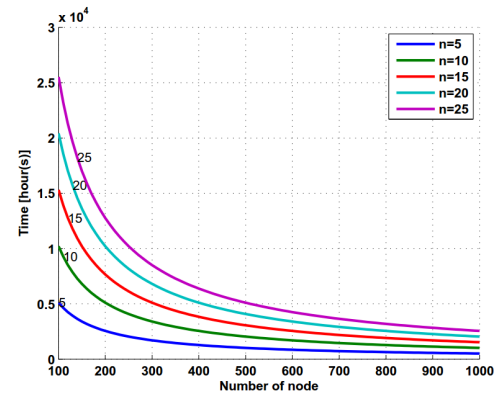

(a) Routed WSN lifetime

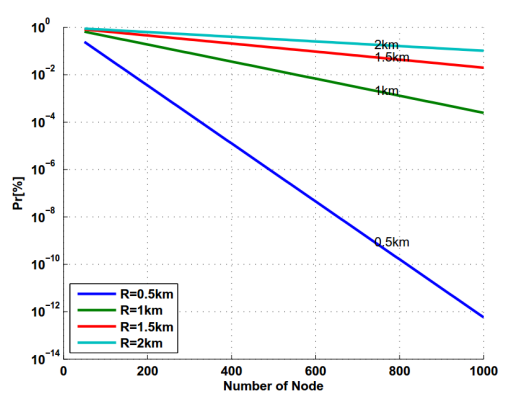

(b) Fraction of uncovered area

Fig. 7. Performance comparison of the poposed and conventional designs.

Consider now a lifetime of a routed WSN. Nodes are assumed to be connected forming a network and all the data are routed to the sinks. Thus, sink nodes will be the first to run out of power. The lifetime of sink nodes depends on the number of nodes in a network, $M$, the number of sink nodes, $n$, and the data collection interval, $d_{t}$. The lifetime of a routed WSN can be approximated as

$$
T_{f}=\frac{T_{c}}{T_{d}} d_{t}=\frac{T_{C} N d_{t}}{t_{C} M} .
$$

Assume that the routed WSN use BLE with the data exchange event consuming $24.762 \mathrm{~mW}$. The lifetime of a routed WSN is shown in Fig. 7(a). Observe that the maximum lifetime of a routed WSN with the same communication technology ( $n=25, M=100, d_{t}=10 \mathrm{~s}$ ) is approximately 2552 hours (106 days) which is 18 times smaller than 48482 hours (2020 days) for the proposed design.

\subsection{Network coverage}

Consider the number of nodes needed to cover a monitored area in the proposed system. The analysis relies upon the results of [7] and is based on integral geometry approach involving, particularly, the notion of kinematic density.

Let $A_{0}\left(F_{0}, L_{0}\right)$ be the monitored area with perimeter $L_{0}$ and area $F_{0}$. Assume $N$ sensors are distributed according to $K\left(A_{0}\right)$ distribution over $A_{0}$. Each sensor has a sensing area $A_{i}\left(F_{i}, L_{i}\right), i=1,2, \ldots, N$, where $L_{i}$ and $F_{i}$ are the perimeter and area, respectively. We are interested in the following metrics: (i) the fraction of area of $A_{0}$ that is not covered, and (ii) the probability that a randomly selected point of $A_{0}$ is covered by at least $k, k \geq 1$ sensors. Assuming that $A_{0}$ and $A_{i}$ are convex, the fraction of area $A_{0}$ that is not covered by any sensors when $N$ sensors are randomly and uniformly deployed is given by [7]

$$
F r(S=0)=\prod_{i=1}^{N} \frac{2 \pi F_{0}+L_{0} L_{i}}{2 \pi\left(F_{0}+F_{i}\right)+L_{0} L_{i}} .
$$




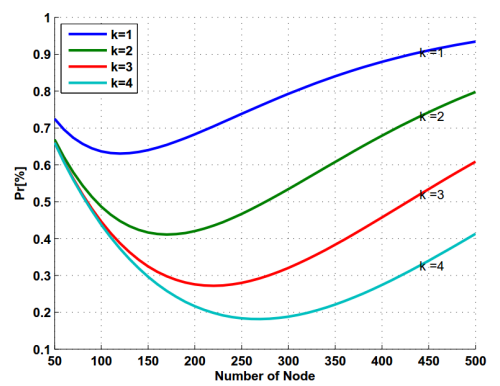

(a) Area radius $1 \mathrm{~km}$

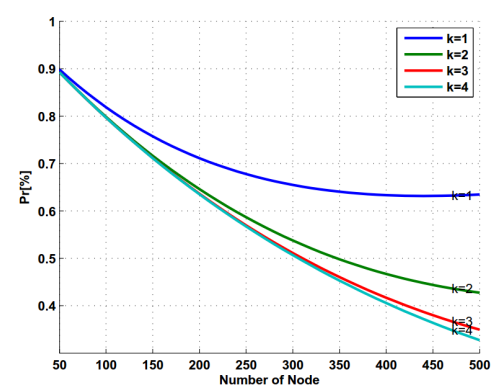

(b) Area radius $2 \mathrm{~km}$

Fig. 8. Probability of an arbitrary point covered by at least $k$ sensors.

The probabability that a point is covered by at least $k, k \geq 1$ sensors is [7]

$$
\operatorname{Pr}\{S \geq k\}=1-\sum_{h=0}^{k-1} \frac{\sum_{i}^{C_{h}^{N}}\left(\prod_{j=1}^{N}\left(2 \pi F_{T_{i, j}}\right) \prod_{z=1}^{N-h} \Theta(i, z)\right)}{\prod_{r=1}^{N}\left(2 \pi\left(F_{0}+F_{r}\right)+L_{0} L_{r}\right)},
$$

where, $\Theta(i, z)=2 \pi F_{0}+L_{0} L_{G_{i, z}}, T_{i, j}$ is a matrix whose each row $i$ is a $k$ permutation of $[1,2 \ldots, N], G_{i, z}$ is a matrix whose each row $i$ contains the elements of $[1,2, \ldots, N]$ that do not appear in the $i$ th row of $T_{i, z}$. The notation $C_{h}^{N}$ denotes binomial coefficient.

The fraction of uncovered area as a function of the number of uniformly deployed nodes is shown in Fig. 7(b). The sensing radius of nodes are assumed to be $100 \mathrm{~m}$ while the monitoring area radii are $0.5,1,1,5,2 \mathrm{~km}$. The fraction of uncovered area decreases exponentially when the number of nodes increases. Using these data one can estimate the number of nodes required to cover a certain area such that only a given small fraction on area is unmonitored.

Fig. 8 illustrates the probability that an arbitrary point of an area is covered by at least $k$ sensors as a function of the number of nodes for different area radii. The redundancy added attempting to cover the largest possible fraction is significant. For example, recalling that for area radius of $1 \mathrm{~km} 365$ nodes cover $95 \%$ of area, we see that this amount of nodes results in non-negligible probability of having two and more nodes covering an arbitrary point of an area. This is a usual price to pay for simplicity of stochastic deployment.

\subsection{Connectivity}

In routed WSN nodes have to be connected. This may require denser deployment than that needed to ensure a coverage only. Here, we evaluate the nodes density required to ensure $k$ connectivity and compare it to that dictated by the coverage.

We approach the connectivity problem using the results of random graph theory. In his seminal paper [10], M. Penrose addressed the question of the distribution of longest edge in a random geometric graph and $k$-connectivity 


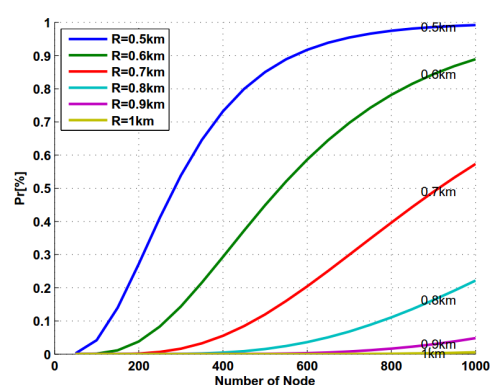

(a) Linear scale

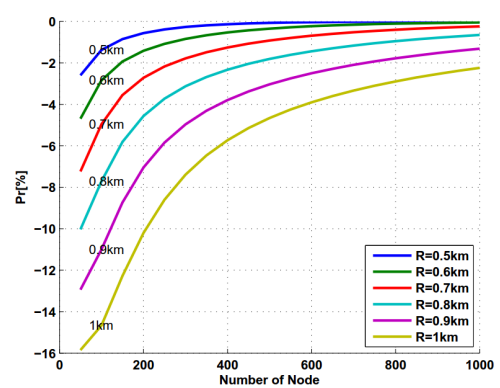

(b) Logarithmic scale

Fig. 9. Probability of 1-connectivity.

of these graphs, respectively. Assuming uniform distribution of nodes over the monitored area and knowing the communications range of a node the former result immediately gives the probability that the network is disconnected while the latter one provides quantities such as the probability of exactly or at least $k$ paths between two randomly chosen nodes.

Let $R$ be the radius of a circular area, $d$ be the communication range of a sensor, $n$ be number of nodes and $k$ be the number of neighbors of arbitrary node. The probability that an arbitrary node has at least $k$ neighbors is [10]

$$
Q_{n, k}(d)=2 \pi \int_{0}^{C} r f(r)\left(1-\sum_{i=0}^{k-1} C_{i}^{n-1} p(r, d)^{i}\left(1-p(r, d)^{n-1-i}\right)\right) d r,
$$

where $f(r)$ is the density of node location, see [6].

The probability that a network with $n$ nodes is $k$-connected is [10]

$$
C_{n, k}(d) \approx\left[Q_{n, k}(d)\right]^{n} .
$$

Fig. 9 shows the probability that the network is connected (1-connected) as a function of the number of deployed nodes for different radius of an area in both linear and log scales. A routed WSN requires much more nodes than the proposed design and the reason is the need for connectivity of the system. Recall that for the proposed WSN design, for an area radius of $1 \mathrm{~km}$ and sensing radius of $100 \mathrm{~m}$, the number of nodes ensuring $95 \%$ coverage is 365 . For routed WSN with nodes having communication range of $100 \mathrm{~m}$, to keep the network connected with 0.95 probability, we need to deploy more than 1000 of nodes. Deploying 365 nodes randomly, the probability of connected network is $6.4 E-7$.

\section{Conclusions}

We proposed and evaluated a new UAV-assisted WSN design. It is based on BLE wireless technology for single-hop communications and UAVs for collection of 
data. The proposed system allows to enforce even energy consumption in WSNs, improves the network lifetime and is still completely automatic in terms of data collection. Based on the modern BLE and UAV specifications, we developed a single-hop sensor-UAV interaction model estimating the optimal altitude and flying speed of UAV for the worst case BLE transaction time as well as the energy required to establish reliable communications.

We analyzed the performance of the proposed design in terms of the lifetime and coverage properties. We also compared these metrics to those of conventional routed WSN design revealing that (i) the proposed system provides 10 to 18 times longer lifetimes compared to conventional WSN and (ii) the density of nodes required to ensure coverage is approximately two times smaller compared to routed WSNs even when sensing region of nodes coincides with their communications range. These properties make the proposal an attractive option for monitoring environmental parameters in large open areas.

\section{References}

1. Akyildiz, I., Kasimoglu, I.: Wireless sensor and actor networks: Research challenges. Ad Hoc Netw. 2, 351-367 (2004)

2. Andreev, S., Gonchukov, P., Himayat, N., Koucheryavy, Y., Turlikov, A.: Energy efficient communications for future broadband cellular networks. Comp. Comm. 35(14), 1662-1671 (2012)

3. Andreev, S., Koucheryavy, Y., Himayat, N., Gonchukov, P., Turlikov, A.: Activemode power optimization in ofdma-based wireless networks. In: Proc. GLOBECOM. pp. 799-803 (2010)

4. Horneber, J., Hergenroder, A.: A survey on testbeds and experimentation environments for wireless sensor networks. IEEE Comm. Surveys and Tutor. 16(4), 1820-1838 (2014)

5. Kamath, S., Lindh, J.: Measuring bluetooth low energy power consumption. Application Note, Texas Instruments (2012)

6. Lassila, P., Hyytia, E., Koskinen, H.: Connectivity properties of random waypoint mobility model for ad hoc networks. In: In IFIP International Federation for Information Processing. pp. 159-168 (2006)

7. Lazos, L., Poovendran, R.: Stochastic coverage in heterogeneous sensor networks. ACM Trans. Sensor Netw. 2(3), 325-358 (2006)

8. Leng, J.: Using a UAV to effectively prolong wireless sensor network lifetime with wireless power transfer. PhD dissertation, University of Nebraska (2014)

9. Moltchanov, D., Koucheryavy, Y., Harju, J.: Simple, accurate and computationally efficient wireless channel modeling algorithm. In: Proc. WWIC. pp. 234-245 (2005)

10. Penrose, M.: The longest edge of the random minimal spanning tree. Ann. Appl. Prob. 7(2), 340-361 (1997)

11. Tuna, G., Mumcu, T., Gulez, K., Gungor, V., Erturk, H.: Unmanned aerial vehicleaided wireless sensor network deployment system for post-disaster monitoring. In: Springer Intelligent Computing Technology and Applications. pp. 298-305 (2012)

12. Valente, J., Sanz, D., Barrientos, A., Cerro, J., Ribeiro, A., Rossi, C.: Anair-ground wireless sensor network for crop monitoring. Sensors 11(6), 6088-6108 (2011)

13. Vinel, A., Vishnevsky, V., Koucheryavy, Y.: A simple analytical model for the periodic broadcasting in vehicular ad-hoc networks. In: Proc. GLOBECOM. pp. $1-5(2008)$ 